\title{
Cedrol induces autophagy and apoptotic cell death in A549 non-small cell lung carcinoma cells through the P13K/Akt signaling pathway, the loss of mitochondrial transmembrane potential and the generation of ROS
}

\author{
SHI-YI ZHANG ${ }^{1}$, XUE-BO LI ${ }^{1}$, SHENG-GUANG HOU ${ }^{2}$, YAO SUN ${ }^{1}$, YI-RAN SHI $^{3}$ and SONG-SEN LIN ${ }^{1}$ \\ Departments of ${ }^{1}$ Radiotherapy and ${ }^{2}$ Pharmacy, Weifang Yidu Central Hospital, Weifang Medical University, Weifang, \\ Shandong 262500; ${ }^{3}$ Department of Surgical Oncology, Weifang People's Hospital, Weifang, Shandong 261041, P.R. China
}

Received June 30, 2015; Accepted April 4, 2016

DOI: $10.3892 / \mathrm{ijmm} .2016 .2585$

\begin{abstract}
The objective of the present study was to determine the anticancer effects of cedrol in A549 human non-small cell lung cancer cells by examining the effects of cedrol on apoptosis induction, the phosphatidylinositol 3'-kinase (PI3K)/Akt signaling pathway, autophagy, reactive oxygen species (ROS) generation and mitochondrial transmembrane potential (MTP). The anticancer effects of cedrol were examined using A549 human lung carcinoma cells as an in vitro model. Cell viability was determined using MTT and lactate dehydrogenase (LDH) assays, and an inverted phase contrast microscope was used to examine the morphological changes in these cells. Cedrol-triggered autophagy was confirmed by transmission electron microscopy (TEM) analysis of the cells, as well as by western blot analysis of microtubule-associated protein lightchain 3 (LC3)B expression. Intracellular ROS generation was measured by flow cytometry using 5-(6)-carboxy-2',7'-dichlorodihydrofluorescein diacetate $\left(\mathrm{CM}-\mathrm{DCFH}_{2}-\mathrm{DA}\right)$ staining and MTP was measured using flow cytometry. The results demonstrated that cedrol reduced cell viability and induced cell apoptosis in a dose-dependent manner. Mechanistic evaluations indicated that cedrol induced apoptosis by reducing the MTP and by decreasing the levels of phosphorylated (p-)PI3K and p-Akt. Cedrol induced autophagy, which was confirmed by TEM analysis, by increasing intracellular ROS formation in a concentration-dependent manner, which was almost completely reversed by $\mathrm{N}$-acetyl-L-cysteine (NAC) and tocopherol. Taken together, these findings reveal that cedrol inhibits cell proliferation and induces apoptosis in A549 cells through mitochondrial and PI3K/Akt signaling pathways. Our
\end{abstract}

Correspondence to: Dr Shi-Yi Zhang, Department of Radiotherapy, Weifang Yidu Central Hospital, Weifang Medical University, 4138 Linglong South Road, Qingzhou, Weifang, Shandong 262500, P.R. China

E-mail: zhangshiyi01@gmail.com

Key words: cedrol, apoptosis, autophagy, lung cancer, reactive oxygen species findings also reveal that cedrol induced pro-death autophagy by increasing intracellular ROS production.

\section{Introduction}

Lung cancer, also known as pulmonary carcinoma, is the most commonly diagnosed type of cancer worldwide accounting for over 1.37 million deaths annually. The activation of oncogenes or the inactivation of tumor suppressor genes have been implicated in the pathogeneis of lung cancer. Mutations in the K-ras proto-oncogene are responsible for $10-25 \%$ of lung adenocarcinomas $(1,2)$. In China, lung cancer has replaced liver cancer as the principal cause of mortality in individuals with malignant tumors. According to the statistics from the National Office on Tumor Cure and Prevention, approximately 600,000 individuals die of lung cancer each year in China (3). Clinically, there are two major types of lung cancer: non-small cell lung carcinoma (NSCLC) and small-cell lung carcinoma (SCLC). NSCLC is more prevalent, accounting for almost $80 \%$ of lung cancers (4).

There are different options for the treatment of NSCLC including chemotherapy with platinum complexes such as cisplatin (cis-diamminedichloroplatinum II) and carboplatin [cis-diammine (1,1-cyclobutane dicarboxylato) platinum II]. The chemotherapy treatment for lung cancer is dependent on the type of tumor. SCLC is primarily treated with chemotherapy and radiotherapy $(5,6)$. However, the long-term administration of chemotherapy may result in drug resistance through gene mutations or DNA methylation. Consequently, the incidence of drug resistance in cancer patients is gradually increasing and anticancer drugs, including paclitaxel, cisplatin, 5-fluorouracil and doxorubicin, are becoming less effective (7). Due to this issue of drug-resistant cancer cells, there is a pressing need to design and develop novel anticancer drugs which are capable of overcoming drug resistance. Plant-based natural products have been an encouraging source of such agents and approximately $60 \%$ of the drugs used clinically for cancer chemotherapy are directly or indirectly derived from plants (8).

Autophagy, the process of self-degradation of redundant or dysfunctional cellular organelles and proteins, is vital for the survival, differentiation, development and homeostasis of living cells. Malfunctioning autophagy plays a significant 
role in the pathogenesis of a number of diseases, including infections, cancer, neurodegenerative disorders, aging, Crohn's disease and heart diseases (9-11). Autophagy may be induced in response to extra- or intracellular stress as well as to signals such as starvation, growth factor deprivation, endoplasmic reticulum (ER) stress and infection by a pathogen (12). Mounting evidence indicates that autophagy plays multiple roles in cancer. Autophagy may act as a tumor suppressor during the initial phases of tumorigenesis by preventing the accumulation of damaged proteins and organelles. It may also act as a tumor promoter through a mechanism which promotes the survival and the growth of tumor cells under conditions of metabolic stress or exposure to therapy $(13,14)$. The intricate autophagy signaling pathways provide potentially significant targets for anticancer therapy $(15,16)$. Anticancer drugs targeted at inducing autophagic cell death, inhibiting protective autophagy or speeding up the transformation of protective autophagy to apoptosis comprise the key practical research strategies currently available. Thus, the role of autophagy in the anticancer effect exerted by natural products has been widely explored in recent years $(17,18)$.

In the present study, we aimed to determine the anticancer effects of cedrol, a plant-derived, oxygenated sesquiterpene, on the NSCLC cell line A549. For this purpose, we explored the role of cedrol in the induction of autophagy and apoptotic cell death through the phosphatidylinositol 3'-kinase (PI3K)/Akt signaling pathway, the loss of mitochondrial transmembrane potential (MTP) and the generation of reactive oxygen species (ROS).

\section{Materials and methods}

Chemicals and antibodies. Cedrol (>98\%) was purchased from Chengdu Pufei De Biotech Co., Ltd. (Chengdu, China). $\mathrm{N}$-acetyl-L-cysteine (NAC) and tocopherol, were purchased from Beyotime Biotech (Jiangsu, China). Specific antibodies against GAPDH (D16H11), microtubule associated protein light-chain 3 (LC3)B [(D11)-\#3868], unc-51 like autophagy activating kinase 1 [ULK1; (D9D7)-\#6439], p70S6K (\#9204), phosphorylated (p-)mTOR (Ser2448), p-ULK1 (Ser317; (D2B6Y)12753), p-P70S6K (Thr421/Ser424) and secondary antibodies (anti-rabbit IgG, HRP-linked antibody; \#7074) were purchased from Cell Signaling Technology (Danvers, MA, USA). RIPA lysis buffer was obtained from Santa Cruz Biotechnology (Santa Cruz, CA, USA). 5-(6)-carboxy-2',7'dichlorodihydrofluorescein diacetate (CM-DCFH $-\mathrm{DA})$, propidium iodide (PI), trypsin and dimethyl sulfoxide (DMSO) were purchased from Sigma-Aldrich (St. Louis, MO, USA). 3-[4,5-Dimethyl-2-thiazolyl]-2,5-diphenyltetrazolium

bromide (MTT), was purchased from Molecular Probes (Eugene, OR, USA). Fetal bovine serum (FBS), and penicillinstreptomycin were obtained from Hangzhou Sijiqing Biological Products Co., Ltd., (Hangzhou, China). Phospho-Akt [p-Akt, Ser-473; (D9E) \#4060], phospho-PI3K (p-PI3K), PI3K, cytochrome $c$ [(136F3) \#4280] and Akt antibodies were purchased from Cell Signaling Technology. $\beta$-actin as well as secondary anti-mouse and anti-rabbit antibodies were purchased from Santa Cruz Biotechnology. Annexin V-FITC apoptosis detection kits and mitochondrial sensor kits containing JC-1 were purchased from Nanjing KeyGen Biotechnology Co., Ltd. (Nanjing, China).
Cell line. The human NSCLC cell line A549 was purchased from the American Type Culture Collection (ATCC; Manassas, VA, USA) and was maintained at $37^{\circ} \mathrm{C}$ in a humidified atmosphere containing $5 \% \mathrm{CO}_{2}$. The cells were cultured in Dulbecco's modified Eagle's medium (DMEM; Sigma-Aldrich) supplemented with $5 \%$ FBS, $2 \%$ penicillin/streptomycin and $2 \%$ amphotericin B (Sigma-Aldrich).

Cell proliferation assay. The effects of cedrol on A549 cell viability were examined by performing an MTT assay. The cells $\left(2 \times 10^{5}\right.$ cells/well in $100 \mu 1$ medium $)$ were seeded into 96 -well plates for $24 \mathrm{~h}$ prior to drug treatment. Taxol (Sigma-Aldrich), which exhibits activity against a number of types of cancer cells, was used as a positive control. Following treatment with various concentrations of cedrol $(0,2.5,5,10$, 20, 40 and $80 \mu \mathrm{M}$ ) for 24,48 and $72 \mathrm{~h}$, the cell plates were treated with MTT solution [10 $\mu \mathrm{l} ; 5 \mathrm{mg} / \mathrm{ml}$ in phosphate-buffered saline (PBS) solution] for an additional $4 \mathrm{~h}$ at $37^{\circ} \mathrm{C}$. The formazan crystals in the viable cells were solubilized with DMSO (150 $\mu \mathrm{l})$ and the absorbance was measured on a microplate reader (ELX 800; Bio-Tek Instruments, Inc., Winooski, VT, USA) at a wavelength of $490 \mathrm{~nm}$. The effects of cedrol on cell viability were calculated as an inhibition ratio (I\%) using the following equation [optical density at $490 \mathrm{~nm}\left(\mathrm{OD}_{490}\right)$ ]:

$$
\mathrm{I} \%=\frac{\left[\mathrm{OD}_{490}(\text { Control })-\mathrm{OD}_{490}(\text { Treated })\right]}{\mathrm{OD}_{490}(\text { Control })} \times 100
$$

Determination of lactate dehydrogenase $(\mathrm{LDH})$ release. The A549 cells were seeded into 96-well plates and treated with cedrol for $24 \mathrm{~h}$, and then $10 \mu \mathrm{LDH}$ release reagent was added to each well. After $1 \mathrm{~h}$, the cell culture plate was centrifuged at $400 \mathrm{x}$ g for $10 \mathrm{~min}$ and $120 \mu \mathrm{l}$ supernatant was removed from each well and transferred into a new black 96-well plate. Next, $60 \mu 1$ of LDH assay mixture was added to each well, and the plate was incubated at $37^{\circ} \mathrm{C}$ for $30 \mathrm{~min}$. The absorbance was measured spectrophotometrically at a wavelength of $490 \mathrm{~nm}$.

Evaluation of A549 cell morphology following cedrol treatment. The A549 cells were plated in 6-well plates at a density of $1 \times 10^{5}$ cells $/ \mathrm{ml}$ and then cultured for $24 \mathrm{~h}$ to facilitate total attachment to the surface of the plates. Subsequently, the cells were subjected to treatment with various concentrations of cedrol $(0$, $5,20$ or $40 \mu \mathrm{M})$ for $48 \mathrm{~h}$. Following drug treatment, the culture plates were examined under an inverted light microscope (Nikon Corporation, Tokyo, Japan) and images were captured.

Measurement of intracellular ROS generation. Intracellular ROS generation was evaluated using fluorescent $\mathrm{CM}_{-\mathrm{DCFH}}$-DA. The A549 cells were seeded into 6-well plates and following adhesion, the cells were pre-treated with $10 \mu \mathrm{M}$

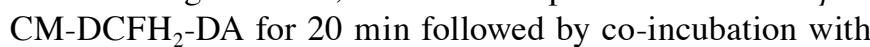
various concentrations of cedrol for another $2 \mathrm{~h}$ and washed with ice-cold PBS twice. The cells were collected and analyzed using a flow cytometer (FACSCanto ${ }^{\mathrm{TM}}$; Becton-Dickinson, Franklin Lakes, NJ, USA) with wavelengths of excitation and emission at 488 and $525 \mathrm{~nm}$, respectively. To explore the role of NAC and tocopherol in the cedrol-induced production of ROS, NAC (2.5 mM) and tocopherol $(10 \mu \mathrm{M})$ were incubated with the A549 cells for $1 \mathrm{~h}$ prior to exposure to cedrol and ROS production was measured. 
A

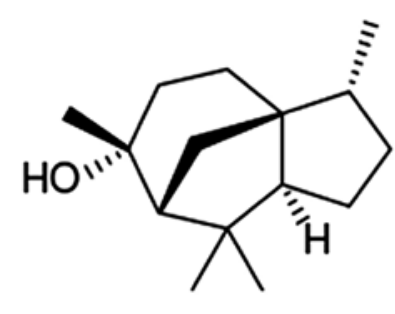

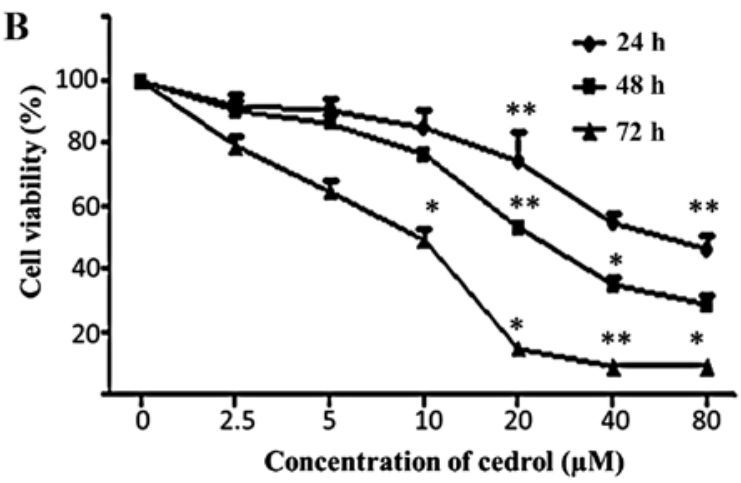

Figure 1. Cedrol inhibits the proliferation of A549 lung cancer cells. (A) The chemical structure of cedrol. (B) A549 cells were treated with various concentrations of cedrol for 24,48 and $72 \mathrm{~h}$. Cell viability was evaluated by performing an MTT assay. Each point is the mean \pm SEM of three experiments. ${ }^{*} \mathrm{P}<0.05$ and ${ }^{* *} \mathrm{P}<0.01$ vs. control group.

Ultrastructural studyofcedrol-induced autophagybytransmission electronic microscopy (TEM). In order to confirm whether autophagy is induced by cedrol treatment in the A549 cancer cells, the cells were treated with cedrol at various concentrations $(0,5,20$ and $40 \mu \mathrm{M})$ for $24 \mathrm{~h}$ and the autophagosomes were visualized using TEM (TEM-1400 plus; JEOL USA, Inc., Peabody, MA, USA) as previously described [Zhou et al, 2014 (19)]. The cells were fixed in ice-cold $2.5 \%$ glutaraldehyde in PBS overnight at $4^{\circ} \mathrm{C}$. The fixed samples were washed three times in PBS. After fixation, the samples were post-fixed in $1 \%$ osmium tetroxide containing $0.1 \%$ potassium ferricyanide for $1 \mathrm{~h}$, and then subjected to TEM analysis.

Western blot analysis. The expression of various proteins in the cells was evaluated using western blot analysis. Briefly, after the cells were treated with cedrol, the total proteins were extracted with RIPA lysis buffer containing $1 \%$ cocktail and 1\% phenylmethanesulfonylfluoride (PMSF). Protein concentrations were determined using a BCA protein assay kit (Pierce Biotechnology, Inc., Rockford, IL, USA). Equivalent amounts of proteins were separated by $10-12 \%$ SDS-PAGE and transferred onto a PVDF membrane (Bio-Rad Laboratories, Hercules, CA, USA). After blocking with 5\% nonfat milk in TBST (10 mM Tris- $\mathrm{HCl}, 500 \mathrm{mM} \mathrm{NaCl}$, and $0.1 \%$ Tween-20) at room temperature for $1 \mathrm{~h}$, the membrane was incubated with specific primary antibodies $(1: 1,000)$ overnight at $4^{\circ} \mathrm{C}$. After washing with TBST, the membrane was incubated with the corresponding secondary antibodies $(1: 10,000)$. To probe for $\mathrm{p}$-PI3K, Akt, p-Akt and $\beta$-actin, the membrane was incubated overnight at $4^{\circ} \mathrm{C}$ with the relevant antibodies. After washing with TBST three times, the membrane was incubated with alkaline phosphatase-conjugated goat anti-rabbit secondary antibodies at room temperature for $1 \mathrm{~h}$ followed by washing with TBST buffer three times. Signals were detected using an ECL Plus Chemiluminescence kit (Applygen Technologies, Inc., Beijing, China) on X-ray film. One hundred and seventy-three gray levels were analyzed using Gel-Pro 32 software (Media Cybernetics, Inc., Rockville, MD, USA).

Evaluation of apoptosis by flow cytometry using an Annexin V-FITC apoptosis detection kit. Apoptosis was evaluated using an Annexin V-FITC apoptosis detection kit. Briefly, the A549 cells were seeded into 6-well plates, incubated over- night, and then treated with 5,20 or $40 \mu \mathrm{M}$ of cedrol for $48 \mathrm{~h}$. The cells were harvested by trypsinization, washed twice with pre-chilled PBS $\left(4^{\circ} \mathrm{C}\right)$ and centrifuged at $251 \mathrm{x} \mathrm{g}$ for $10 \mathrm{~min}$. The cell pellet was resuspended in binding buffer $(400 \mu \mathrm{l})$ and the rate of apoptosis was examined using Annexin V-FITC and PI double staining according to the manufacturer's instructions. After staining the cells with Annexin V-FITC/PI, flow cytometric analysis was performed using a FACSCalibur flow cytometer (Becton-Dickinson), and the data were analyzed using CellQuest software.

Determination of MTP using flow cytometry. MTP was measured using JC-1 staining. Briefly, the A549 cells were seeded into 6-well plates and incubated without (control) or with $(5,20$ or $40 \mu \mathrm{M})$ of cedrol for $48 \mathrm{~h}$. After incubation, the cells were collected, and the cell pellets were washed twice with ice-cold PBS and then resuspended in DMEM at a density of $1 \times 10^{6}$ cells $/ \mathrm{ml}$. The cells were then incubated with JC-1 $(1 \mu \mathrm{l})$ at $37^{\circ} \mathrm{C}$ for $15 \mathrm{~min}$. The stained cells were washed twice with PBS, resuspended in PBS (500 $\mu \mathrm{l})$ and analyzed using flow cytometry with excitation and emission wavelengths of 488 and $530 \mathrm{~nm}$, respectively. The fluorescence of the treated cells was compared with the untreated control group.

Statistical analysis. Data are presented as the means \pm SEM of the control. All experiments were repeated at least three times. The differences between groups were analyzed by one-way ANOVA with Tukey's post hoc tests. $\mathrm{P}<0.05$ and $\mathrm{P}<0.01$ was considered to indicate a statistically significant difference.

\section{Results}

Cedrol inhibits the proliferation of A549 lung cancer cells. The MTT assay was used to examine the inhibitory effects of cedrol on the proliferation of A549 cells. The chemical structure of cedrol is shown in Fig. 1A. The results indicated that cedrol markedly inhibited the proliferation of the human lung cancer cell line A549 (Fig. 1B). Furthermore, time- and concentration-dependent inhibition curves were observed in these cells. The calculated $\mathrm{IC}_{50}$ values at 24,48 and $72 \mathrm{~h}$ were $31.87 \pm 9.3,14.54 \pm 2.18$ and $5.02 \pm 0.7 \mu \mathrm{M})$, respectively. In addition, $\mathrm{LDH}$ release into the culture medium was also increased in a concentration-dependent manner (Fig. 2). The results of 


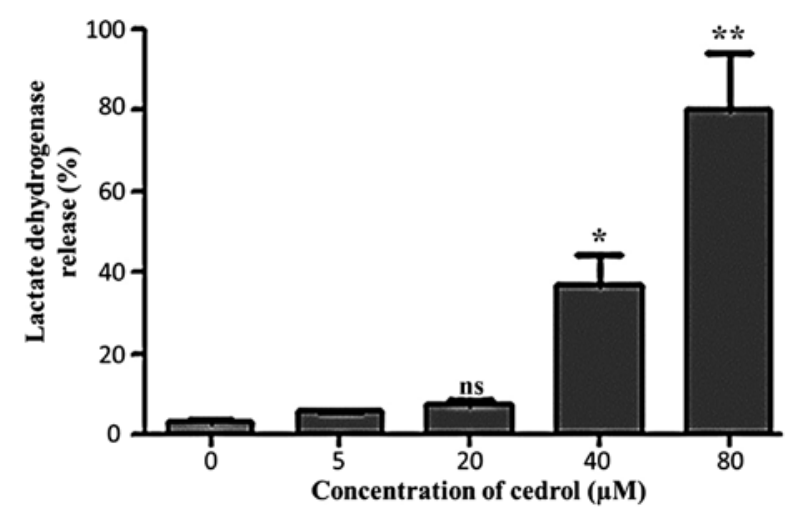

Figure 2. Cedrol increases lactate dehydogenase (LDH) release from A549 cells. The cells were treated with various concentrations of cedrol for $24 \mathrm{~h}$ and LDH release was detected using an LDH assay kit. Each point is the mean \pm SEM of three experiments. " $\mathrm{P}<0.05$ and ${ }^{* *} \mathrm{P}<0.01$ vs. control group. ns, non-significant. these two assays indicated that cedrol induced potent cytotoxic effects in the A549 cells in a dose-dependent manner.

Phase contrast microscopic evaluation of the cedrol-induced cell cytotoxicity. The exposure of A549 cells to 5, 20 or $40 \mu \mathrm{M}$ of cedrol for $48 \mathrm{~h}$ resulted in a substantial decrease in cell counts and furthermore, induced morphological changes that were characteristic of cytotoxicity (cell shrinking, loss of membrane integrity) in the A549 cells. The cells were examined under an inverted phase contrast microscope following exposure to cedrol (Fig. 3).

Cedrol induces ROS formation in A549 cells. The effect of cedrol on intracellular ROS production was measured by flow cytometry using a fluorescent probe $\mathrm{CM}-\mathrm{DCFH}_{2}$-DA. As shown in Fig. 4A and B, ROS formation was markedly
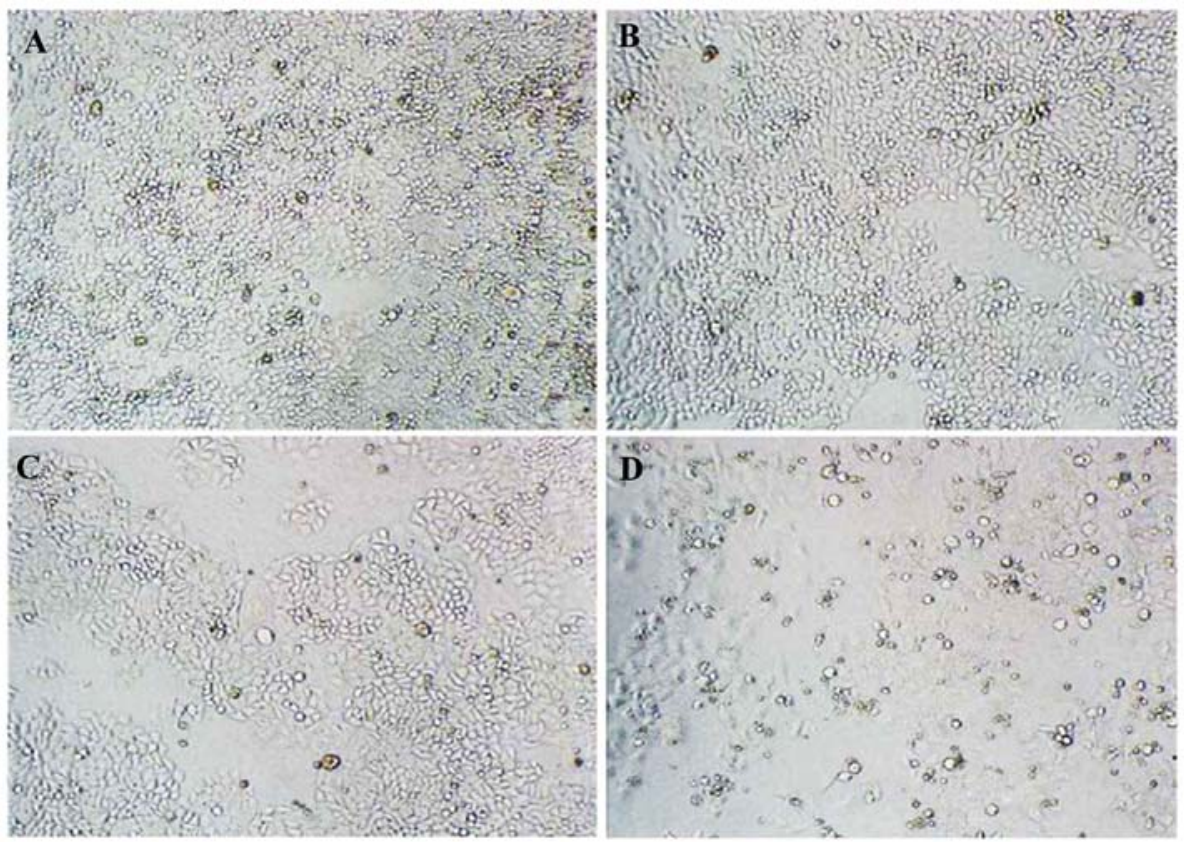

Figure 3. Effects of cedrol on the morphological characteristics of A549 cells. Morphological changes in the A549 cells were observed under a phase-contrast microscope after treating the cells (A) without [control] or (B) with 5, (C) 20 and (D) $40 \mu \mathrm{M}$ of cedrol for $48 \mathrm{~h}$; magnification, x10.

A
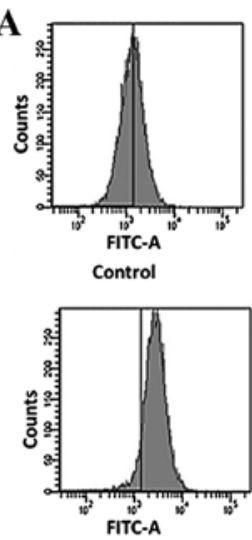

$20 \mu \mathrm{M}$
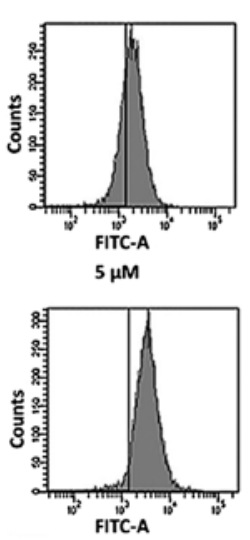

$40 \mu \mathrm{M}$

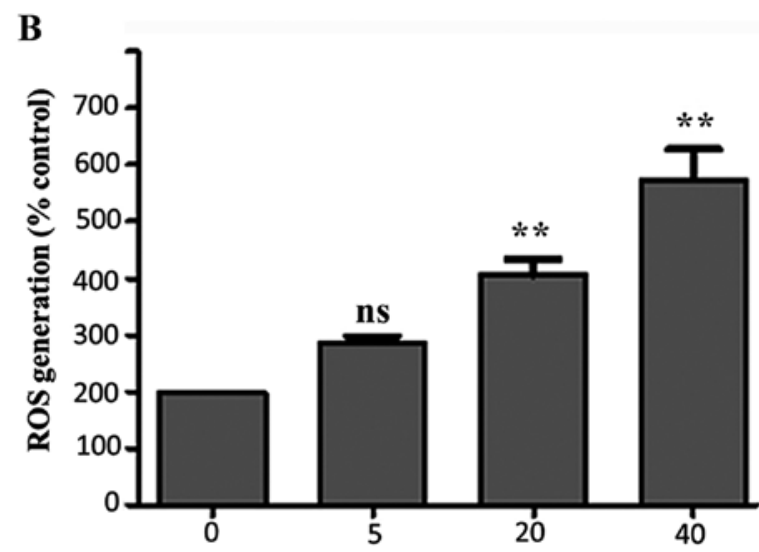

Concentration of cedrol $(\mu \mathrm{M})$

Figure 4. Cedrol induces reactive oxygen species (ROS) generation in A549 cells. (A and B) A549 cells were treated with cedrol (0, 5, 20 and 40 mM) for 2 h, and ROS generation was evaluated using $\mathrm{CM}-\mathrm{DCFH}_{2}$-DA staining and flow cytometry. ${ }^{* *} \mathrm{P}<0.01$ vs. control group; ns, non-significant. 


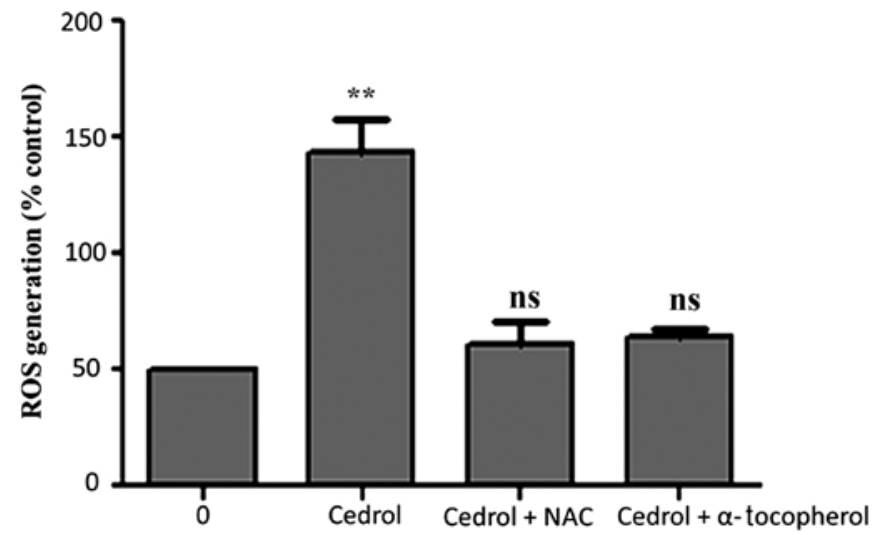

Figure 5. A549 cells were treated with N-acetyl-L-cysteine (NAC) (2.5 mM) or tocopherol $(10 \mu \mathrm{M})$ for $1 \mathrm{~h}$ prior to treatment with cedrol $(40 \mu \mathrm{M})$. Reactive oxygen species (ROS) production was detected using CM-DCFH $\mathrm{CHA}_{2}$ staining. ${ }^{* *} \mathrm{P}<0.01$ vs. control group; ns, non-significant.

induced after treating the A549 cells with cedrol for $2 \mathrm{~h}$. ROS generation occurred in a concentration-dependent manner and a two-fold increase in ROS production was observed following treatment with $20 \mu \mathrm{M}$ cedrol. In addition, pre-treatment with NAC $(2.5 \mathrm{mM})$ or tocopherol $(10 \mu \mathrm{M})$ for $1 \mathrm{~h}$ almost completely reversed the cedrol-induced generation of ROS in the A549 cells (Fig. 5).

Cedrol induces the autophagic cell death of A549 cells. Cedrol-induced autophagy was first examined using TEM. TEM analysis revealed that compared with the control group (Fig. 6A), cedrol treatment $(5,20$ and $40 \mu \mathrm{M})$ induced a large number of autophagosomes (Fig. 6B-D) and this number increased with increasing concentrations of cedrol. Cedrolinduced autophagy was also evaluated by western blot analysis. The results revealed that cedrol treatment induced a substantial increase in the protein expression of LC3B in the A549 cells in a dose-dependent manner (Fig. 7A). In addition, the results of western blot analysis indicated that cedrol treatment markedly suppressed the protein expression of p-mTOR and p-P70S6K and increased the protein expression of ULK1 and p-ULK1 in the A549 cells in a concentration-dependent manner (Fig. 7B).

Cedrol induces the apoptosis of A549 cells . To confirm whether cedrol-induced cell death in A549 cells may be mediated through the activation of the apoptotic cascade, the cells were treated with different concentrations of cedrol, stained with Annexin V-FITC/PI and analyzed for apoptosis using flow cytometry. We observed a dose-dependent increase in the percentage of apoptotic cells $(2.02,18.3,29.61$ and $53.41 \%$ following $48 \mathrm{~h}$ of treatment with $0,5,20$ or $40 \mu \mathrm{M}$ of cedrol, respectively) (Fig. 8). The results indicated that cedrol efficiently suppressed cell growth and induced apoptosis in human lung cancer cells in vitro.

Cedrol induces the apoptosis of A549 cells through the PI3K/Akt signaling pathway. The PI3K/Akt signaling pathway is recognized to be one of the key signaling pathways involved in the regulation of cell growth, proliferation and apoptosis. Akt is a major downstream target of PI3K, and Akt phosphorylation leads to the significant upregulation of Bcl-2 expression, and the downregulation of Bax expression. We examined the effects of cedrol on the PI3K/Akt signaling pathway by measuring the protein levels of p-PI3K, total Akt, and p-Akt. It was observed that cedrol significantly downregu-
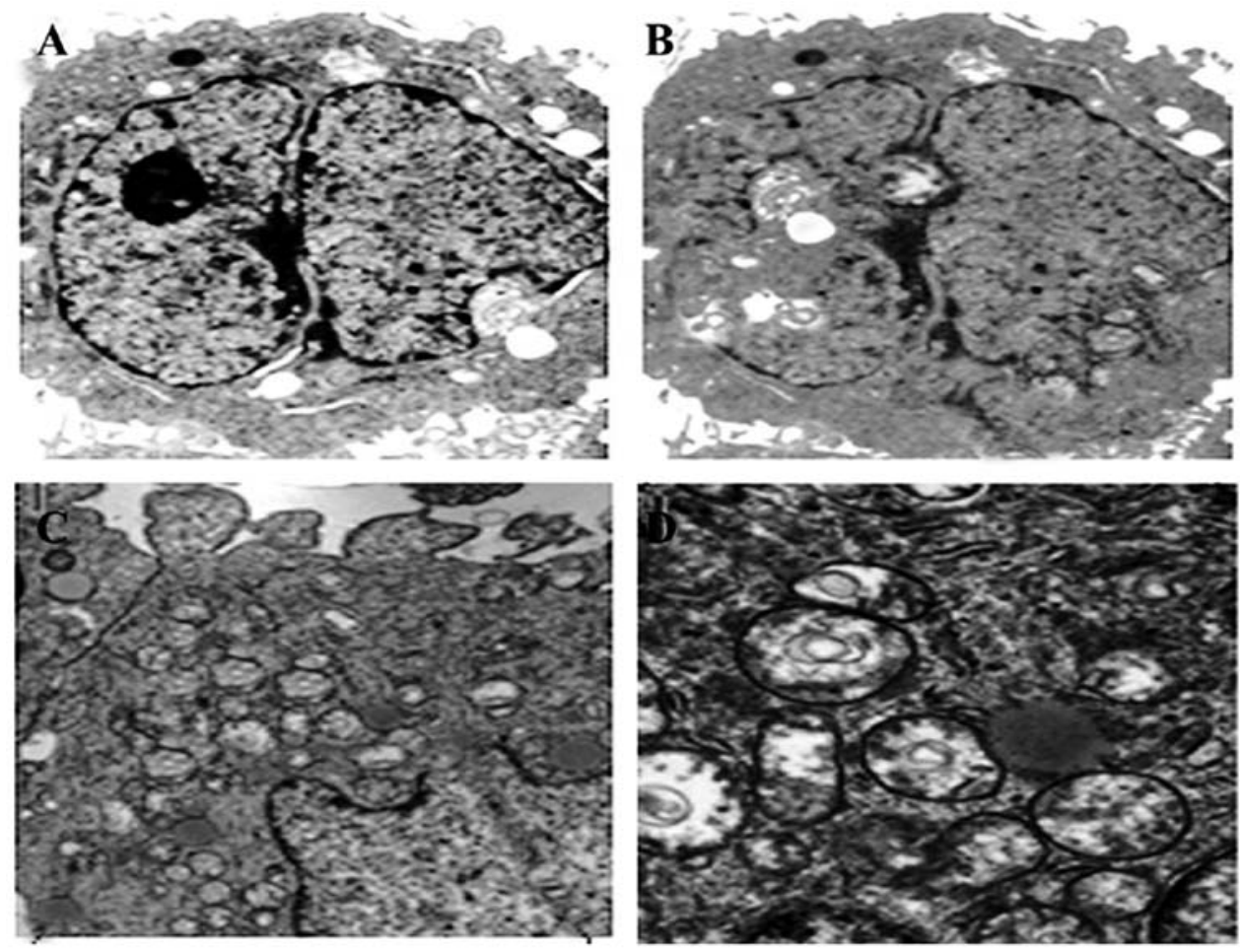

Figure 6. Cedrol, at concentrations of (A) 0,(B) 5, (C) 20 and (D) $40 \mathrm{mM}$, induces autophagosomes in A549 cells. Images were captured using transmission electron microscopy after a $24 \mathrm{~h}$ treatment period; magnification, $\mathrm{x} 40$. 
A

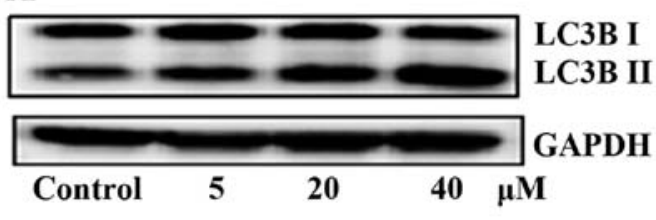

B

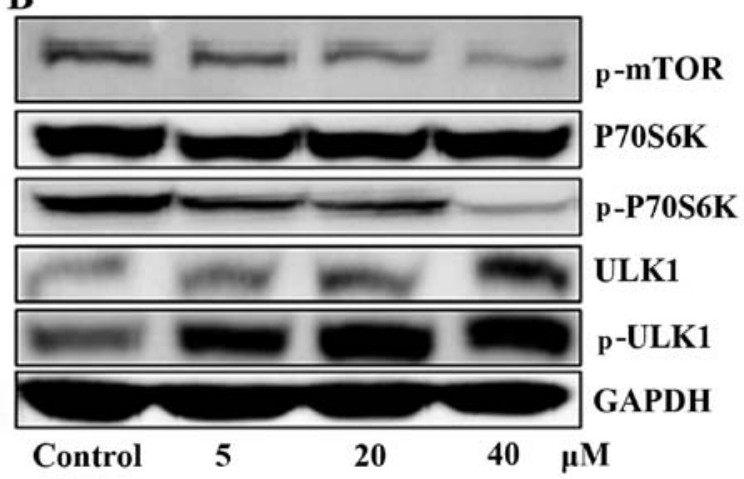

Figure 7. Cedrol induces autophagy in A-549 lung cancer cells. The cells were treated with cedrol (0, 5, 20 and $40 \mathrm{mM})$ for $24 \mathrm{~h}$, respectively. (A) Cell lysates were prepared and subjected to western blot analysis to evaluate the protein expression of microtubule-associated protein light-chain 3 (LC3)B. (B) A549 cells were treated with cedrol $(0,5,20$ and $40 \mu \mathrm{M})$ for $24 \mathrm{~h}$, and the expression of autophagic proteins was evaluated using western blot analysis. ULK1, unc-51 like autophagy activating kinase 1 .
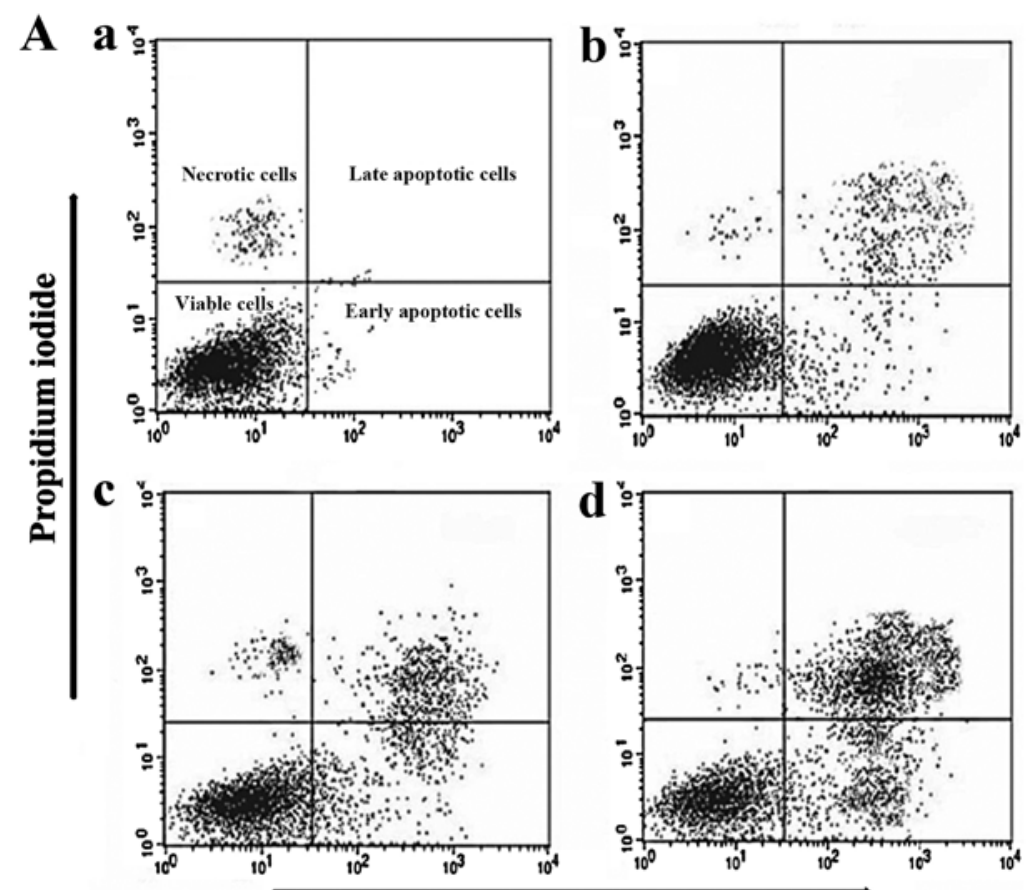

Annexin V-FITC

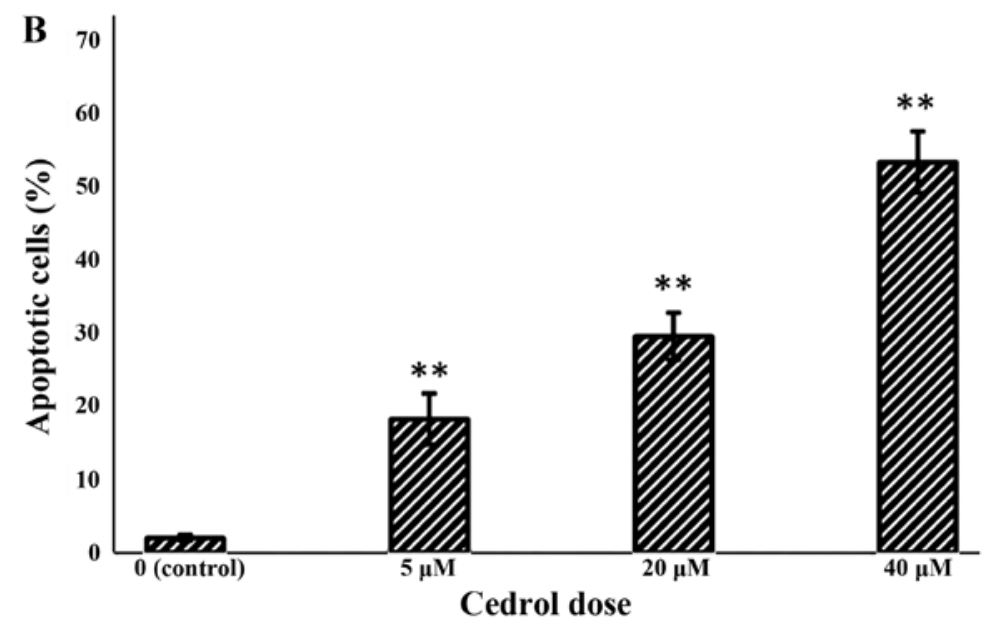

Figure 8. Cedrol induces apoptosis in A-549 cells at different concentrations. (A, panels a to d) Annexin V/PI double-staining assay of A549 cells. The cell

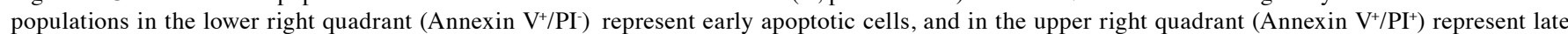
apoptotic cells. Panels a to d represent the control, 5, 20 and $40 \mu \mathrm{M}$ cedrol-treated cells, respectively. (B) The apoptotic rates of A549 cells induced by $0,5,20$ and $40 \mu \mathrm{M}$ of cedrol for $48 \mathrm{~h}$. Data are presented as the means $\pm \mathrm{SD}(\mathrm{n}=3){ }^{* * *} \mathrm{P}<0.01$ as compared with control cells. 

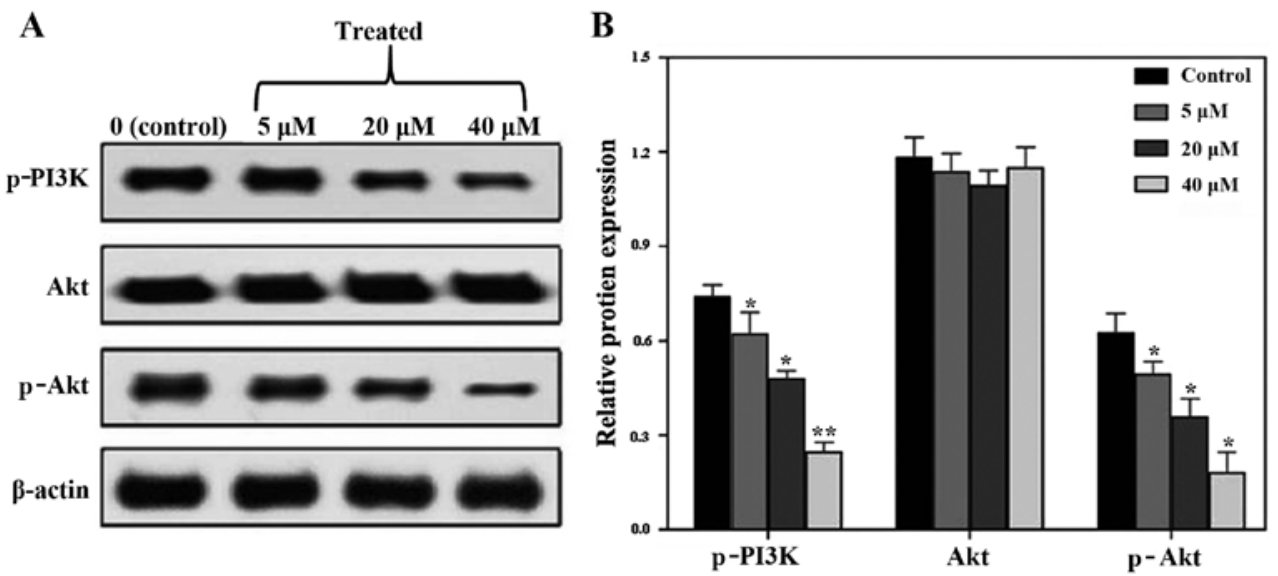

Figure 9. Effects of cedrol on the protein expression of apoptosis-related proteins. A549 cells were treated with 0, 5, 20 and $40 \mu \mathrm{M}$ of cedrol for $48 \mathrm{~h}$, after which the cell lysates were extracted and subjected to western blot analysis in order to determine the expression of phosphorylated (p-)PI3K, Akt and p-Akt (A) Representative blots are shown. (B) The optical densities of the bands were measured using Gel-Pro 32 software and data are presented as the means \pm SD of at least three independent experiments.
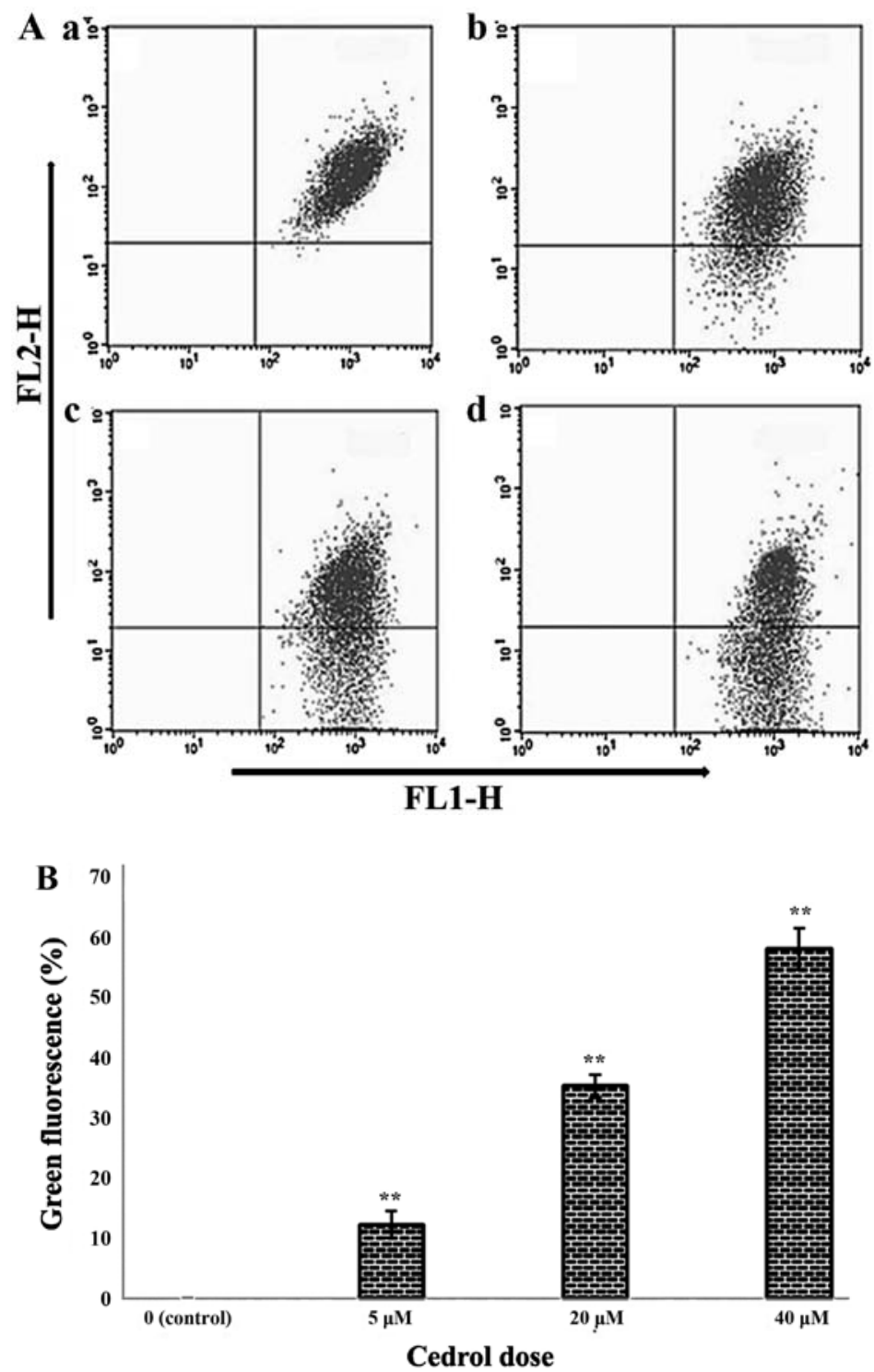

Figure 10. Cedrol decreases the mitochondrial transmembrane potential (MTP) in A549 cells. (A) Cells were exposed to cedrol (panels a to d, 0, 5, 20 and $40 \mu \mathrm{M}$, respectively) for $48 \mathrm{~h}$ and the MTP was measured using JC-1 staining in conjuction with flow cytometry. The cell populations in the upper right quadrant (red fluorescence of JC-1 dimers) represent high MTP, and in the lower right quadrant (green fluorescence of JC-1 monomers) represent low MTP. (B) The values indicate the percentage of green fluorescence (JC-1 monomers) in the A549 cells treated without or with cedrol for $48 \mathrm{~h}$. Data are presented as the means $\pm \mathrm{SD}(\mathrm{n}=3) .{ }^{* * *} \mathrm{P}<0.01$ as compared with control cells. 
lated the expression of p-Akt and p-PI3K in the A549 cells in a dose-dependent manner whereas the total Akt protein levels remained constant during all treatments (Fig. 9).

Cedrol decreases MTP in A549 cells. Mitochondria are important organelles that are involved in the release of apoptotic signals during an intrinsic pathway for the execution of apoptosis (20). Mitochondrial dysfunction leads to a reduction of the MTP as well as the release of cytochrome $c$ from the mitochondria into the cytosol (21), which is one of the mechanisms through which caspase activation induces apoptotic cell death. To determine the effects of cedrol on MTP, the A549 cells were treated with cedrol for $48 \mathrm{~h}$, and MTP was measured using JC-1 staining in conjunction with flow cytometry. This assay is based on the principle that the red fluorescent JC-1 dimers will be present in areas with high MTP (upper-right quadrant of the fluorescence cytogram), whereas green fluorescent JC-1 monomers will be prevalent in areas with low MTP (lower-right quadrant of the fluorescence cytogram). As shown in Fig. 10, green fluorescence increased from 0.25 to $55.92 \%$ as the concentration of cedrol increased. The results indicated that cedrol-induced apoptosis was caused by mitochondrial dysfunction induced by a reduction in MTP.

\section{Discussion}

Natural products have been increasingly used as anticancer agents in recent decades. Natural products have played an important role in the design and development of $>60 \%$ of the anticancer drugs in clinical use $(8,22)$. In addition, there are several natural products or their analogs which are currently undergoing preclinical and clinical testing. The World Health Organization has determined that about $75-80 \%$ of the world population depend on traditional medicines for their primary health care needs (8). Natural products, which are highly effective and possess fewer side-effects, are a promising substitute for chemotherapy associated with deleterious side-effects (23). Non-cytotoxic, bioactive molecules have great potential applications for use in the treatment of brain tumors as the majority of these natural products exhibit pleiotropic properties (24). Cedrol is a sesquiterpene alcohol which is found in many essential oils, including cedar, cypress and juniper oils. Cedrol comprises approximately $15-19 \%$ of cedarwood oil (25).

The PI3K/Akt signaling pathway is an important signal transduction pathway which has been reported to play crucial roles in suppressing apoptosis and enhancing cell proliferation. This pathway is known to affect the activation of a series of downstream effector molecules. The PI3K/Akt pathway has been implicated in a large number of human malignancies (26-29). The results of our study indicated that cedrol significantly downregulated the expression of p-Akt and $\mathrm{p}-\mathrm{PI} 3 \mathrm{~K}$ in the A549 cells in a dose-dependent manner whereas the total Akt protein levels remained constant during all treatments.

Autophagy is a process of self-degradation of unimportant or non-functional cellular organelles and proteins, and plays a key role in the pathogenesis of a number of diseases including cancer. In the present study, we also examined whether cedrol induces autophagy in A549 lung cancer cells using TEM and western blot analysis. The results indicated that cedrol induced a large number of autophagosomes in these cells and this number increased with increasing concentrations of cedrol. Furthermore, western blot analysis revealed that cedrol treatment induced a significant increase in the protein expression of LC3B in the A549 cells in a dose-dependent manner. In addition, cedrol treatment significantly blocked the protein expression of p-mTOR and p-P70S6K, and increased the protein expression of ULK1 and p-ULK1.

Apoptosis is the other mode of cell death through which a cell self-destructs and is a highly organized and complex process. As cell death may involve either necrosis or apoptosis, it is important to evaluate the mode of cell death induced by cedrol treatment. Unlike necrosis, apoptosis is a process of programmed cell death and does not involve inflammatory responses (30). The results of our study indicated that cedrol treatment led to apoptotic cell death in the A549 lung cancer cells; there was a dose-dependent increase in the percentage of apoptotic cells $(2.02,18.3,29.61$ and $53.41 \%$ following a $48 \mathrm{~h}$ treatment period with $0,5,20$ or $40 \mu \mathrm{M}$ of cedrol, respectively) was observed. In addition, cedrol treatment increased ROS generation in a dose-dependent manner. Pre-treatment with NAC or tocopherol for $1 \mathrm{~h}$ almost completely reversed the cedrol-induced production of ROS in the A549 cells.

In conclusion, cedrol exerts anticancer effects in A549 human lung cancer cells which are mediated through apoptosis, autophagy, the PI3K/Akt signaling pathway, ROS generation and the loss of MTP. All these effects need to be investigated using different in vivo models and further PK-PD studies are warranted to fully examine toxicity profiles in order to confirm whether cedrol is an effective therapeutic agent for the treatment of non-small cell lung carcinoma.

\section{References}

1. Jemal A, Siegel R, Xu J and Ward E: Cancer statistics, 2010. CA Cancer J Clin 60: 277-300, 2010.

2. Ferlay J, Shin HR, Bray F, Forman D, Mathers C and Parkin DM: Estimates of worldwide burden of cancer in 2008: GLOBOCAN 2008. Int J Cancer 127: 2893-2917, 2010.

3. Liu Q and Zhou Q: The challenges of lung cancer in China. J Cancer Res Ther 9 (Suppl 2): S65-S66, 2013.

4. Butnor KJ and Beasley MB: Resolving dilemmas in lung cancer staging and histologic typing. Arch Pathol Lab Med 131: 1014-1015, 2007.

5. Hann CL and Rudin CM: Management of small-cell lung cancer: incremental changes but hope for the future. Oncology (Williston Park) 22: 1486-1492, 2008.

6. Goffin J, Lacchetti C, Ellis PM, Ung YC and Evans WK; Lung Cancer Disease Site Group of Cancer Care Ontario's Program in Evidence-Based Care: First-line systemic chemotherapy in the treatment of advanced non-small cell lung cancer: a systematic review. J Thorac Oncol 5: 260-274, 2010.

7. Rebucci M and Michiels C: Molecular aspects of cancer cell resistance to chemotherapy. Biochem Pharmacol 85: 1219-1226, 2013.

8. Newman DJ and Cragg GM: Natural products as sources of new drugs over the 30 years from 1981 to 2010. J Nat Prod 75: 311-335, 2012.

9. Levine B and Kroemer G: Autophagy in the pathogenesis of disease. Cell 132: 27-42, 2008.

10. Murrow L and Debnath J: Autophagy as a stress-response and quality-control mechanism: implications for cell injury and human disease. Annu Rev Pathol 8: 105-137, 2013.

11. He C and Klionsky DJ: Regulation mechanisms and signaling pathways of autophagy. Annu Rev Genet 43: 67-93, 2009.

12. Choi KS: Autophagy and cancer. Exp Mol Med 44: 109-120, 2012. 
13. Yang ZJ, Chee CE, Huang S and Sinicrope FA: The role of autophagy in cancer: therapeutic implications. Mol Cancer Ther 10: 1533-1541, 2011.

14. Janku F, McConkey DJ, Hong DS and Kurzrock R: Autophagy as a target for anticancer therapy. Nat Rev Clin Oncol 8: 528-539, 2011.

15. Kung CP, Budina A, Balaburski G, Bergenstock MK and Murphy M: Autophagy in tumor suppression and cancer therapy. Crit Rev Eukaryot Gene Expr 21: 71-100, 2011.

16. Mizushima N, Levine B, Cuervo AM and Klionsky DJ: Autophagy fights disease through cellular self-digestion. Nature 451: 1069-1075, 2008.

17. Ding Q, Bao J, Zhao W, Hu Y, Lu J and Chen X: Natural autophagy regulators in cancer therapy: A review. Phytochem Rev 14: 137-154, 2014.

18. Zhou M and Wang R: Small-molecule regulators of autophagy and their potential therapeutic applications. ChemMedChem 8 : 694-707, 2013

19. Zhou T, Ye L, Bai Y, Sun A, Cox B, Liu D, Li Y, Liotta D, Snyder JP, Fu H and Huang B: Autophagy and apoptosis in hepatocellular carcinoma induced by EF25-(GSH)2: a novel curcumin analog. PLoS One 9: e107876, 2014.

20. Ohta S: A multi-functional organelle mitochondrion is involved in cell death, proliferation and disease. Curr Med Chem 10 2485-2494, 2003.

21. Kakkar P and Singh BK: Mitochondria: a hub of redox activities and cellular distress control. Mol Cell Biochem 305: 235-253, 2007.
22. Kingston DG: Modern natural products drug discovery and its relevance to biodiversity conservation. J Nat Prod 74: 496-511, 2011.

23. Bent $\mathrm{S}$ and Ko R: Commonly used herbal medicines in the United States: a review. Am J Med 116: 478-485, 2004.

24. Wagner $\mathrm{H}$ and Ulrich-Merzenich G: Synergy research: approaching a new generation of phytopharmaceuticals. Phytomedicine 16: 97-110, 2009.

25. Connolly JD and Hill RA (eds): Dictionary of Terpenoids. Volume 1: Mono- and Sesquiterpenoids. Chapman \& Hall, London, 1991.

26. Jücker M, Südel K, Horn S, Sickel M, Wegner W, Fiedler W and Feldman RA: Expression of a mutated form of the p85alpha regulatory subunit of phosphatidylinositol 3-kinase in a Hodgkin's lymphoma-derived cell line (CO). Leukemia 16: 894-901, 2002.

27. Xu Q, Simpson SE, Scialla TJ, Bagg A and Carroll M: Survival of acute myeloid leukemia cells requires PI3 kinase activation. Blood 102: 972-980, 2003.

28. Levine DA, Bogomolniy F, Yee CJ, Lash A, Barakat RR, Borgen PI and Boyd J: Frequent mutation of the PIK3CA gene in ovarian and breast cancers. Clin Cancer Res 11: 2875-2878, 2005.

29. Oda K, Stokoe D, Taketani Y and McCormick F: High frequency of coexistent mutations of PIK3CA and PTEN genes in endometrial carcinoma. Cancer Res 65: 10669-10673, 2005.

30. de Bruin EC and Medema JP: Apoptosis and non-apoptotic deaths in cancer development and treatment response. Cancer Treat Rev 34: 737-749, 2008. 